\title{
DAMIT: a database of asteroid models
}

\author{
J. Durech ${ }^{1}$, V. Sidorin ${ }^{2}$, and M. Kaasalainen ${ }^{3}$ \\ 1 Astronomical Institute, Faculty of Mathematics and Physics, Charles University in Prague, V Holešovičkách 2, \\ 18000 Prague, Czech Republic \\ e-mail: durech@sirrah.troja.mff.cuni.cz \\ 2 Astronomical Institute, Academy of Sciences, Boční II, 14131 Prague, Czech Republic \\ 3 Tampere University of Technology, PO Box 553, 33101 Tampere, Finland
}

Received 15 June 2009 / Accepted 20 January 2010

\begin{abstract}
Context. Apart from a few targets that were directly imaged by spacecraft, remote sensing techniques are the main source of information about the basic physical properties of asteroids, such as the size, the spin state, or the spectral type. The most widely used observing technique - time-resolved photometry - provides us with data that can be used for deriving asteroid shapes and spin states. In the past decade, inversion of asteroid lightcurves has led to more than a hundred asteroid models. In the next decade, when data from all-sky surveys are available, the number of asteroid models will increase. Combining photometry with, e.g., adaptive optics data produces more detailed models.

Aims. We created the Database of Asteroid Models from Inversion Techniques (DAMIT) with the aim of providing the astronomical community access to reliable and up-to-date physical models of asteroids - i.e., their shapes, rotation periods, and spin axis directions. Models from DAMIT can be used for further detailed studies of individual objects, as well as for statistical studies of the whole set. Methods. Most DAMIT models were derived from photometric data by the lightcurve inversion method. Some of them have been further refined or scaled using adaptive optics images, infrared observations, or occultation data. A substantial number of the models were derived also using sparse photometric data from astrometric databases.

Results. At present, the database contains models of more than one hundred asteroids. For each asteroid, DAMIT provides the polyhedral shape model, the sidereal rotation period, the spin axis direction, and the photometric data used for the inversion. The database is updated when new models are available or when already published models are updated or refined. We have also released the $\mathrm{C}$ source code for the lightcurve inversion and for the direct problem (updates and extensions will follow).
\end{abstract}

Key words. minor planets, asteroids: general - astronomical data bases: miscellaneous

\section{Introduction}

The knowledge of basic physical characteristics of asteroids the spin rate, the spin axis orientation, the shape, the size, the mass, the spectral type, etc. - is important for understanding the history and the current state of the asteroid population. Apart from a few asteroids directly imaged by spacecraft, our knowledge about asteroid physical properties is based on remote sensing techniques. And from all these techniques, time-resolved photometry is by far the most important source of information because it is in principle available for all known asteroids. The lightcurve inversion method developed by Kaasalainen \& Torppa (2001) and Kaasalainen et al. (2001) is a powerful tool for deriving asteroid shapes and spins from their lightcurves observed over several apparitions.

So far, the lightcurve inversion method has led to models of about one hundred asteroids. The models have been published in several papers (Kaasalainen et al. 2002b, 2004; Torppa et al. 2003; Durech et al. 2009, for example). As has been shown by Kaasalainen et al. (2004) or Durech et al. (2005) and demonstrated on real targets by Durech et al. (2009), physical models of asteroids can also be derived from photometry that only sparsely samples brightness variations. This kind of photometric data will be provided by all-sky surveys like Pan-STARRS (Panoramic Survey Telescope and Rapid Response System), LSST (Large Synoptic Survey Telescope), or Gaia (Mignard et al. 2007), and we expect an avalanche of new asteroid models in the next decade.

Models obtained by lightcurve inversion are interesting as such, but their main importance is that, by using the lightcurve inversion, we can reveal new physical phenomena. For example, it was used when the alignment of spins of the Koronis family members was revealed (Slivan et al. 2003) or when the YORP (Yarkovsky-O'Keefe-Radzievskii-Paddack) effect was detected (Kaasalainen et al. 2007). In general, the analysis of spin states and shapes of asteroids has been always related to the inversion of disk-integrated lightcurves (see review papers Magnusson et al. 1989; Pravec et al. 2002, for example). Lightcurve inversion has become a standard tool for revealing asteroids' nature from photometry. Moreover, lightcurves can be combined with other data (Kaasalainen \& Lamberg 2006; Kaasalainen 2009). For example, adaptive optics images can provide us with nonconvex details (Descamps et al. 2009), and thermal infrared observations can determine the correct absolute size of the object, its thermal inertia, and albedo (Delbo \& Tanga 2009). The reliability of models derived from photometry has been proven by comparison with the real shapes revealed by spacecraft (Müller et al. 2005; Lamy et al. 2008) and by laboratory experiments (Kaasalainen et al. 2005).

To provide an easy access to most of the published lightcurve inversion models we set up the Database of Asteroid Models from Inversion Techniques (DAMIT). DAMIT is an MySQL database accessible via a web interface. In the following section, 
we describe the structure of DAMIT and the format and physical meaning of individual database items.

\section{Database description}

The aim of the database is to provide the community access to all published asteroid models derived by the lightcurve and other inversion methods, as well as to source codes for solving direct and inverse problems. For each asteroid in the database, the basic physical parameters are given: the shape model, the spin axis orientation, and the rotation period. DAMIT also contains information about the light-scattering model used in the inversion. The lightcurves from which the model was derived can be downloaded as a data file. Other data sources, such as adaptive optics images, are cited and the data included as auxiliary files when appropriate. The reference to the paper where the model (or its update) was published is always given. However, a number of DAMIT solutions are significantly different from those published in the original papers. This is mainly due to the updated data set or to a range too narrow for the sidereal period search used in the original work. In some cases, photometric data files have been corrupted or misread, and rechecks and data corrections have led to some updates.

In principle, there can be more models for one asteroid, usually because of a limited geometry (an asteroid orbiting too close to the ecliptic, see Kaasalainen \& Lamberg 2006 for details) that introduces an ambiguity $\lambda \pm 180^{\circ}$ in the ecliptic longitude of the pole. Or there can be more models corresponding to a different inversion method being applied, e.g., a convex and a nonconvex model for the same asteroid. So far, DAMIT contains $\sim 180$ models for more than one hundred asteroids. Usually, the models have similar values for the rms residual. If one of the models is preferred - either due to a lower rms residual or for another reason $-\mathrm{a}$ comment is added to it.

\subsection{Shape models}

Asteroid shape models are represented by polyhedrons with triangular surface facets (see an example in Fig. 1). A single file with the list of $x, y, z$ Cartesian coordinates of the polyhedron vertices followed by the list of facets represents each model. Vertices of the corresponding triangle are listed as seen from outside the body in the counterclockwise sense. Because we cannot infer size information from photometry alone, most of the models are scale-free and are arbitrarily scaled to have a unit volume. If a model is scaled to its real dimensions (when, e.g., occultation data, high-angular-resolution data, or infrared measurements are available), this is stated in the corresponding tag, and the $x, y, z$ coordinates are given in kilometres. The model always rotates around its $z$ axis, which is usually close, but not exactly identical, to the maximum principal axis of the inertia tensor (assuming a uniform density distribution). If these two axes differ significantly, the model formally fits the data well, but the rotation around the $z$ axis is physically impossible. Such cases are discussed. There is only one such model in the current database -167 Urda. The $x$ axis has no special orientation with respect to the minimum principal axis of the inertia tensor. It is usually defined implicitly by setting $t_{0}$ in Eq. (1) to the minimum JD epoch in the lightcurve data set (or to the nearest lower integer value).

So far, DAMIT does not contain any model of a "tumbling", asteroid, i.e., an asteroid in an excited rotation state. However, we plan to include such models in the future. The first example will be the model of asteroid 2008 TC3 derived from photometry (Scheirich et al., in prep.). DAMIT also does not contain models of binary asteroids. In cases where the satellite is much smaller than the primary, DAMIT contains models of primary components of binary or multiple systems (87 Sylvia and 121 Hermione, for example). For synchronous binaries, there is only one period in the lightcurves and it should be possible to create a convex model that fits the data well. We usually do not include those asteroids in DAMIT for which a detailed model derived from radar delay-Doppler echoes or from direct spacecraft imaging is available. These high-resolution models can be found in the Planetary Data System (PDS) archive ${ }^{1}$.

Each shape model is visualized and shown from three directions. There are two views from the asteroid's equator and one from its pole. The three views correspond to the views from the positive $x, y, z$ axes, respectively. The light-scattering model used for rendering has no physical meaning and was chosen just for visualization purposes using Matlab software.

The vast majority of DAMIT asteroid shape models are convex. As shown by Durech \& Kaasalainen (2003), disk-integrated lightcurves contain very little information about shape nonconvexities. To reveal nonconvex features from lightcurves, observations at very high phase angles (when shadowing effects are important) are necessary. Moreover, nonconvex solutions lack the stability and uniqueness properties of the convex solutions. Reliable nonconvex shape models can be derived only when lighcurves are combined with high-resolution data (adaptive optics images, for example). This also means that we cannot properly determine the nonconvexity errors of a convex shape model. The match between a convex model and the real shape can be very good for almost-convex bodies (2 Pallas) or can be poor for highly nonconvex bodies (624 Hektor).

The errors of the shape determination were discussed in Kaasalainen \& Torppa (2001) and Kaasalainen et al. (2001). They showed that the formal errors are worthless in practice because the effect of random noise is usually negligible compared to systematic and model errors. The only practical way of estimating real errors is to perform a series of optimizations with different methods, scattering laws, and initial values to see how the results differ. However, such detailed investigation of errors is usually not included in those papers where the shape models are published. In general, shape models are much better constrained in the equatorial plane than in the direction of the pole.

\subsection{Spin}

The rotation state of an asteroid is described by its sidereal rotation period $P$, the fixed orientation of its spin axis (the $z$ axis in the body frame) expressed by means of the ecliptic coordinates $(\lambda, \beta)$, and the initial rotation angle $\phi_{0}$ for some epoch $t_{0}$. The orientation of the model in the inertial frame is then obtained by applying a set of rotations on the model. The transformation from the body-fixed coordinate frame $\boldsymbol{r}_{\text {ast }}$ to the ecliptic coordinate frame $\boldsymbol{r}_{\mathrm{ecl}}$ for the time $t$ is given by the equation

$r_{\mathrm{ecl}}=\mathrm{R}_{z}(\lambda) \mathrm{R}_{y}\left(90^{\circ}-\beta\right) \mathrm{R}_{z}\left(\phi_{0}+\frac{2 \pi}{P}\left(t-t_{0}\right)\right) \boldsymbol{r}_{\mathrm{ast}}$,

where $\mathrm{R}_{i}(\theta)$ is a rotation matrix around $i$ th axis

$$
\mathrm{R}_{z}(\theta)=\left(\begin{array}{ccc}
\cos \theta & -\sin \theta & 0 \\
\sin \theta & \cos \theta & 0 \\
0 & 0 & 1
\end{array}\right), \quad \mathrm{R}_{y}(\theta)=\left(\begin{array}{ccc}
\cos \theta & 0 & \sin \theta \\
0 & 1 & 0 \\
-\sin \theta & 0 & \cos \theta
\end{array}\right)
$$

An example illustrating the four subsequent rotations and the definition of the rotation angles is shown in Fig. 1.

\footnotetext{
1 http://www.psi.edu/pds/
} 

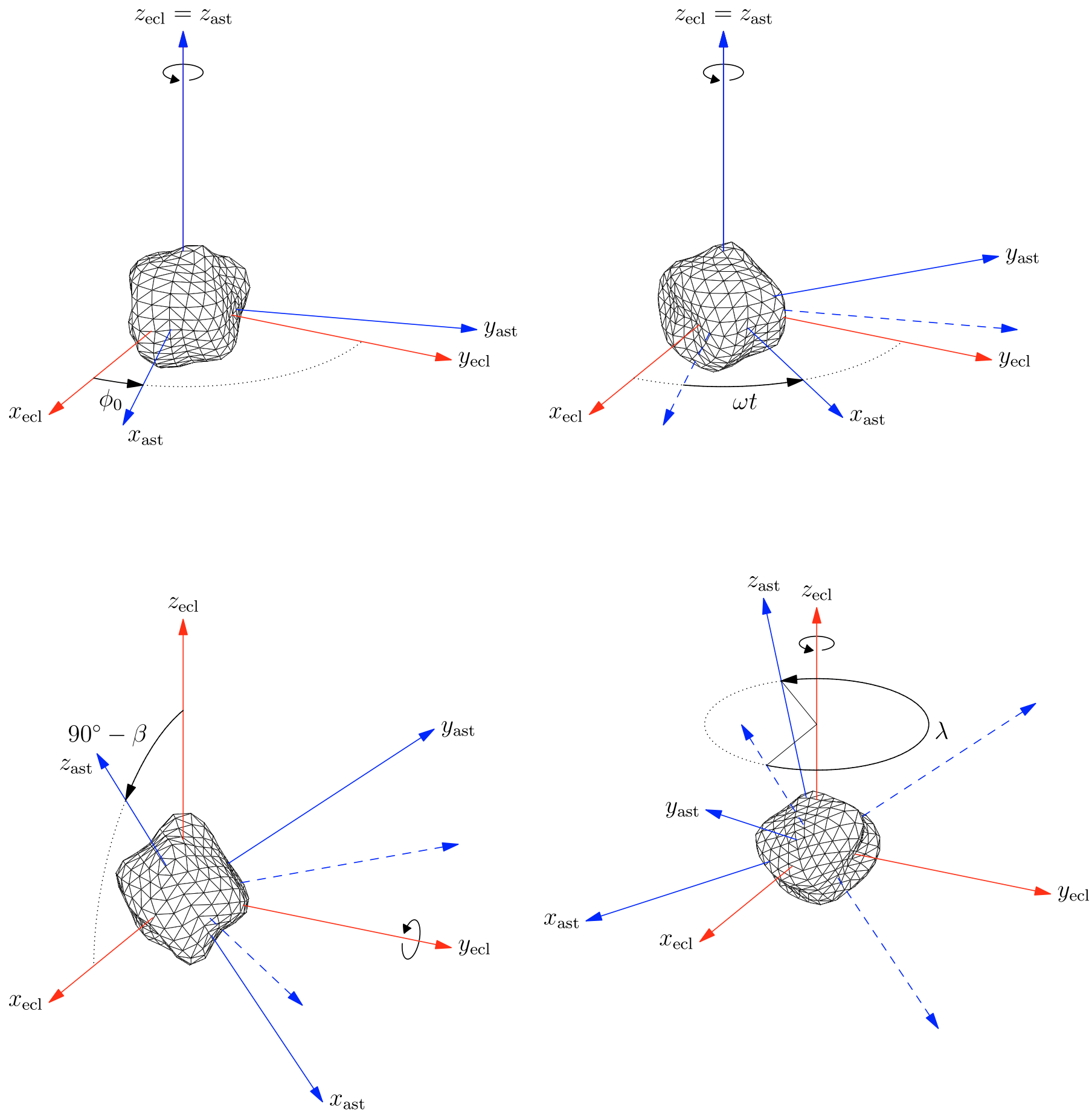

Fig. 1. An example of the rotational sequence described by Eq. (1). The four subsequent rotations transform the model from the body-fixed coordinate frame "ast" to the ecliptic coordinate frame "ecl". Here $\phi_{0}$ is the initial rotation angle, $\omega=2 \pi / P$ is the spin rate, and $\beta$ and $\lambda$ are the ecliptic latitude and longitude of the pole, respectively.

For some asteroids in the database, the rotation period is not constant in time but changes secularly from the YORP effect (Kaasalainen et al. 2007; Durech et al. 2008a). In such cases, the corresponding rotation matrix has the form

$\mathrm{R}_{z}\left(\phi_{0}+\frac{2 \pi}{P_{0}}\left(t-t_{0}\right)+\frac{1}{2} v\left(t-t_{0}\right)^{2}\right)$,

where $v \equiv \mathrm{d} \omega / \mathrm{d} t$ is the linear change of the rotation rate $\omega=$ $2 \pi / P$. The rotation period $P_{0}$ is then given for $t_{0}$.
The orientation of a model given by Eq. (1) is expressed in the asteroid-centric reference frame. When computing, for example, the sky-plane projection of a model for a given time $t_{\mathrm{obs}}$, one has to take the light travel time from the asteroid to the observer into account. Then $t=t_{\mathrm{obs}}-\Delta / c$, where $\Delta$ is the asteroidobserver distance and $c$ the speed of light.

The description of the spin-state given above does not follow the recommendation of the IAU as defined by Seidelmann et al. (2007), mainly because the rotation angles $\lambda$ and $\beta$ in Eq. (1) are (contrary to the equatorial reference frame angles $\alpha$ and $\delta$ 
recommended by the IAU) directly related to the spin-orbital dynamics. For low-inclination asteroids, $90^{\circ}-\beta$ is the obliquity. However, for each model in the database, we also give the rotation elements according to the IAU recommendation: the direction of the positive spin axis in the equatorial coordinates $\alpha$, $\delta$, the rotational rate $\dot{W}$, and the position of the prime meridian $W_{0}$ for time $t_{0}$. The position of the prime meridian $W$ at time $t$ can be computed as $W=W_{0}+\dot{W}\left(t-t_{0}\right)$.

The rotation parameters listed in the database can also be downloaded as a file. This file can be used directly as input for the direct-problem software that enables one to generate lightcurves for a given shape and spin (see Sect. 2.6).

The relative accuracy of the rotation period depends mainly on the total time span of the observations. The higher the number of revolutions in the interval of observations, the higher the accuracy of the length of one revolution, i.e, of the rotation period. The accuracy of the period $P$ corresponds to the order of the last decimal place of $P$. The typical relative error ranges from $10^{-5}$ to $10^{-6}$.

Contrary to the database of asteroid spin vector determinations maintained by Kryszczyńska at the Poznań Observatory (Kryszczyńska et al. 2007) that compiles all available spin vector solutions derived by various techniques, all DAMIT spin vector solutions were derived using one inversion method. The typical uncertainty of the spin axis direction is about $5-10^{\circ}$.

\subsection{Light-scattering model}

Lightcurve inversion models are usually derived under the assumption that the light-scattering behaviour of asteroids can be described as a combination of Lommel-Seeliger and Lambert models (Kaasalainen et al. 2002a). The surface reflectance $S$ as a function of the angle of incidence $i$ and the angle of emergence $e$ is then

$S\left(\mu, \mu_{0}, \alpha\right)=f(\alpha)\left[S_{\mathrm{LS}}\left(\mu, \mu_{0}\right)+c S_{\mathrm{L}}\left(\mu, \mu_{0}\right)\right]$,

where $\mu_{0}=\cos i, \mu=\cos e$, the Lommel-Seeliger term is

$S_{\mathrm{LS}}\left(\mu, \mu_{0}\right)=\frac{\mu \mu_{0}}{\mu+\mu_{0}}$,

the Lambert term is

$S_{\mathrm{L}}\left(\mu, \mu_{0}\right)=\mu \mu_{0}$,

and $c$ is the weight factor. Function $f(\alpha)$ describes the dependence on the solar phase angle $\alpha$ and is expressed by means of three parameters $a, d, k$ as

$f(\alpha)=a \exp \left(-\frac{\alpha}{d}\right)+k \alpha+1$

The parameters $c, a, d$, and $k$ are listed in the database. However, if there are not enough calibrated lightcurves to cover a sufficiently wide range of phase angles, $f(\alpha)$ cannot be derived and parameters $a, d, k$ are missing.

In some cases, when accurate calibrated photometry covering a wide range of phase angle was available, instead of the simple model above, the Hapke scattering model (Hapke 1981, 1984, 1986) was used during the inversion. In such cases, DAMIT lists the five parameters of Hapke's model: the singleparticle scattering albedo $w$, the asymmetry parameter of the single-particle function $g$, the opposition surge amplitude $B_{0}$, the opposition surge width $h$, and the macroscopic roughness $\bar{\theta}$.

\subsection{Lightcurves}

The photometric data used in the inversion can be downloaded from DAMIT as data files. The lightcurve file contains brightness in intensity units for given epochs (light-time corrected) and the viewing and illumination geometry. The Cartesian ecliptic coordinates of the Sun and the Earth with respect to the asteroid are given for each data point.

A tag at the beginning of each lightcurve states whether the lightcurve is relative - i.e., can be shifted on the magnitude scale with respect to other relative lightcurves - or if the brightness measurements are calibrated and no shift with respect to other calibrated curves is allowed. The lightcurve files can be directly used as input for the inversion software (see Sect. 2.6). For each lightcurve, the reference to the original paper where the data were published is given. References to papers in which the original photometric data used for inversion were published are listed in the Appendix A.

\subsection{Realization}

DAMIT is accessible online at http://astro.troja.mff. cuni.cz/projects/asteroids3D. The core of DAMIT is an MySQL database served by the MySQL server, which ensures high stability and reliability, as well as flexibility for future development. The web interface of DAMIT is written in PHP and allows users to either browse or search the database. Records from the database can be downloaded as text files suitable for further processing. The shape solutions are also provided in the form of computer-generated images in PNG (Portable Network Graphics) format. DAMIT with all its components is hosted and operated by the Astronomical Institute of the Charles University. We plan to set up a mirror site to minimize all negative impacts of a possible hardware failure.

\subsection{Software}

DAMIT also contains source codes of inversion programs and a tool for lightcurve generation along with the user's manuals. The formats of input and output files are identical to the formats of lightcurve and shape files in DAMIT. The software is available under the General Public Licence, and we encourage all potential users to use it for inversion of their own data and to develop it further. The software will be described in detail in a forthcoming paper.

\section{Conclusions}

The number of asteroid models derived from lightcurves and other sources by inversion techniques is continuously growing, so we expect a dramatic increase of the number of models in the next few years with the dawn of all-sky surveys. The number of observers who use their own data for shape modelling is also growing, mainly thanks to the availability of the Windows-based user-friendly version of the inversion software ${ }^{2}$. We believe that DAMIT is going to become the main archive for asteroid models and that it will be widely recognized as a reliable and up-to-date source of asteroid spin parameters.

To increase the scientific attractivity of DAMIT, we will connect it with the IMCCE Virtual Observatory Solar System por$\mathrm{tal}^{3}$. The service will compute physical ephemerides of models

\footnotetext{
2 http://wwW.minorplanetobserver.com

3 http://vo.imcce. fr/webservices
} 
stored in DAMIT, for example, sky-plane projections of models for a given epoch with a realistic brightness distribution (Berthier et al. 2008).

Acknowledgements. The work of J.L. was supported by grants GACR 205/07/P070 of the Czech grant agency and by the Research Program MSM0021620860 of the Ministry of Education. The work of MK was supported by the Academy of Finland.

\section{Appendix A: Lightcurve references}

Below we list the references to papers in which the original photometric data used for inversion were published. For some asteroids, DAMIT contains also unpublished lightcurves. USNO stands for the US Naval Observatory in Flagstaff - the photometry obtained at this observatory as a by-product of astrometric observations was downloaded from the AstDys page 4 and used for modelling.

\section{(2) Pallas}

Binzel (1984)

Burchi (1972)

Burchi \& Milano (1983)

Burchi et al. (1985)

Carlsson \& Lagerkvist (1983)

Groeneveld \& Kuiper (1954b)

Chang \& Chang (1963)

Chang et al. (1981)

Lagerkvist et al. (1988)

Lupishko et al. (1989)

Lustig \& Hahn (1976)

Schober et al. (1994)

Schroll et al. (1976)

van Houten-Groeneveld (1981)

Wood \& Kuiper (1963)

\section{(3) Juno}

Birch \& Taylor (1989)

di Martino et al. (1987b)

Gehrels \& Owings (1962)

Groeneveld \& Kuiper (1954b)

Harris et al. (1989a)

Millis et al. (1981)

Schroll et al. (1981)

\section{(5) Astraea}

Gehrels \& Owings (1962)

Harris et al. (1999)

Chang \& Chang (1962)

Lagerkvist et al. (1989)

Melillo (1987a)

Pavlovski et al. (1981)

Taylor (1978)

USNO

Weidenschilling et al. (1990)

\section{(6) Hebe}

Ahmad (1954)

di Martino \& Cacciatori (1984a)

Dotto et al. (1995)

Erikson et al. (1991)

Gehrels \& Taylor (1977)
Hutton \& Blain (1988)

Hainaut-Rouelle et al. (1995)

Lagerkvist et al. (1995)

Wamsteker \& Sather (1974)

Yang et al. (1965)

Zhou et al. (1982)

\section{(7) Iris}

Foglia (1992)

Gehrels \& Owings (1962)

Groeneveld \& Kuiper (1954a)

Chang \& Chang (1963)

Lagerkvist \& Williams (1987)

Taylor (1977)

van Houten-Groeneveld \& van Houten (1958)

Zhou et al. (1982)

\section{(8) Flora}

Ahmad (1954)

Debehogne et al. (1990)

di Martino et al. (1989)

Harris \& Young (1989)

Hollis et al. (1987)

Piironen et al. (1998)

Pilcher (2009)

van Houten-Groeneveld \& van Houten (1958)

Vesely \& Taylor (1985)

Veverka (1971)

Zappala et al. (1983b)

Zhou et al. (1982)

\section{(9) Metis}

di Martino \& Cacciatori (1984a)

di Martino et al. (1987b)

Gehrels \& Owings (1962)

Groeneveld \& Kuiper (1954a)

Groeneveld \& Kuiper (1954b)

Harris \& Young (1989)

Chang \& Chang (1962)

Melillo (1987b)

Schober \& Surdej (1979)

Weidenschilling et al. (1987)

Weidenschilling et al. (1990)

Yang et al. (1965)

Zappala \& van Houten-Groeneveld (1979)

Zeigler \& Florence (1985)

\section{(15) Eunomia}

Foglia (1995)

Groeneveld \& Kuiper (1954a)

Chang et al. (1959)

Chang et al. (1981)

Lagerkvist \& Williams (1987)

Lagerkvist et al. (1989)

Melillo (1986)

Piironen et al. (1985)

Scaltriti \& Zappala (1975)

van Houten-Groeneveld \& van Houten (1958)

Vesely \& Taylor (1985)

Weidenschilling et al. (1987) 


\section{(16) Psyche}

Dotto et al. (1992)

Chang et al. (1981)

Lupishko et al. (1980)

Lupishko et al. (1982)

Lupishko et al. (1989)

Neely (1992)

Taylor et al. (1976)

Tedesco \& Taylor (1985)

Tedesco et al. (1983)

van Houten-Groeneveld \& van Houten (1958)

Weidenschilling et al. (1987)

Zhou \& Yang (1981)

Zhou et al. (1982)

\section{(17) Thetis}

Denchev et al. (1998)

Groeneveld \& Kuiper (1954a)

Harris et al. (1992)

Lagerkvist et al. (1995)

Lupishko et al. (1979)

Lupishko et al. (1989)

Michałowski et al. (1995)

USNO

van Houten-Groeneveld \& van Houten (1958)

\section{(19) Fortuna}

Denchev (2000, private comm.)

Harris et al. (1992)

Harris et al. (1999)

Lagerkvist et al. (1987)

Lupishko et al. (1981)

Lupishko et al. (1989)

van Houten-Groeneveld et al. (1979)

Weidenschilling et al. (1987)

Weidenschilling et al. (1990)

\section{(20) Massalia}

Barucci et al. (1985)

Dotto et al. (1995)

Gehrels (1956)

Gehrels \& Owings (1962)

Hainaut-Rouelle et al. (1995)

Chang \& Chang (1962)

McCheyne et al. (1985)

\section{(21) Lutetia}

Denchev (2000, private comm.)

Denchev et al. (1998)

Dotto et al. (1992)

Chang \& Chang (1963)

Lagerkvist et al. (1995)

Lupishko et al. (1983)

Lupishko et al. (1987a)

Zappala et al. (1984)

\section{(22) Kalliope}

Gehrels \& Owings (1962)

Lupishko et al. (1982)

Lupishko et al. (1989)

Melillo (1987a)

Michałowski \& Velichko (1990)
Scaltriti et al. (1978)

Surdej et al. (1986)

Weidenschilling et al. (1987)

Weidenschilling et al. (1990)

Zappala \& van Houten-Groeneveld (1979)

\section{(23) Thalia}

Hainaut-Rouelle et al. (1995)

Harris \& Young (1983)

Lagerkvist et al. (1995)

Pilcher - unpublished data

Tancredi \& Gallardo (1991)

van Houten-Groeneveld et al. (1979)

Vesely \& Taylor (1985)

Yang et al. (1965)

Ziegler \& Florence (1985)

\section{(29) Amphitrite}

Debehogne et al. (1978)

Chang \& Chang (1963)

McCheyne et al. (1984)

McCheyne et al. (1985)

Polishook (2009)

Tedesco \& Sather (1981)

van Houten-Groeneveld et al. (1979)

Weidenschilling et al. (1987)

\section{(30) Urania}

Gehrels \& Owings (1962)

Harris \& Young (1980)

USNO

Wisniewski et al. (1997)

\section{(32) Pomona}

di Martino et al. (1987b)

Dotto et al. (1995)

Erikson et al. (1991)

Chang et al. (1981)

Schober (1976)

\section{(34) Circe}

Ďurech et al. (2009)

Lagerkvist et al. (1987)

Lagerkvist et al. (1995)

Magnusson \& Lagerkvist (1991)

USNO

Vesely \& Taylor (1985)

\section{(39) Laetitia}

di Martino et al. (1987b)

Gehrels \& Owings (1962)

Groeneveld \& Kuiper (1954a)

Groeneveld \& Kuiper (1954b)

Chang et al. (1981)

McCheyne et al. (1985)

Sather (1976)

van Houten-Groeneveld \& van Houten (1958)

Vesely \& Taylor (1985)

Wamsteker \& Sather (1974)

Weidenschilling et al. (1987)

Weidenschilling et al. (1990) 


\section{(41) Daphne}

Barucci (1983)

Barucci et al. (1985)

Scaltriti \& Zappala (1977b)

Weidenschilling et al. (1987)

Weidenschilling et al. (1990)

\section{(43) Ariadne}

Barucci et al. (1986)

Burchi \& Milano (1974)

di Martino \& Cacciatori (1984a)

di Martino et al. (1987b)

Dotto et al. (1995)

Lustig \& Dvorak (1975)

McCheyne et al. (1984)

Shevchenko et al. (1992)

van Houten-Groeneveld et al. (1979)

Weidenschilling et al. (1987)

Weidenschilling et al. (1990)

\section{(44) Nysa}

Birch et al. (1983)

di Martino et al. (1987b)

Gehrels \& Owings (1962)

Groeneveld \& Kuiper (1954b)

Harris \& Young (1983)

Harris et al. (1989b)

Chang \& Chang (1962)

Shatzel (1954)

Shevchenko et al. (1992)

Taylor \& Tedesco (1983)

Weidenschilling et al. (1990)

Yang et al. (1965)

Zappala \& van Houten-Groeneveld (1979)

\section{(45) Eugenia}

Debehogne \& Zappala (1980)

Hutton (1989)

Harris \& Young (1979)

Harris et al. (1999)

Lebofsky et al. (1988)

Shevchenko et al. (1992)

Tancredi \& Gallardo (1991)

Taylor et al. (1988)

Weidenschilling et al. (1987)

\section{(52) Europa}

Barucci et al. (1986)

Dotto et al. (1995)

Michałowski et al. (1995)

Michałowski et al. (2004)

Scaltriti \& Zappala (1977c)

Zappala et al. (1983a)

\section{(54) Alexandra}

Belskaya et al. (1993)

di Martino et al. (1987b)

Haupt \& Hanslmeier (1985)

Tancredi \& Gallardo (1991)

USNO

van Houten-Groeneveld et al. (1979)

Warner et al. (2008)

\section{(55) Pandora}

Barucci et al. (1985)

di Martino \& Cacciatori (1984a)

di Martino et al. (1987b)

Lagerkvist et al. (1995)

Shevchenko et al. (1993)

Schober (1978)

Weidenschilling et al. (1987)

Weidenschilling et al. (1990)

\section{(63) Ausonia}

di Martino \& Cacciatori (1984a)

Hainaut-Rouelle et al. (1995)

Lagerkvist (1981)

Lagerkvist \& Williams (1987)

Lagerkvist et al. (1989)

Scaltriti \& Zappala (1977a)

Zappala et al. (1983b)

\section{(73) Klytia}

Hainaut-Rouelle et al. (1995)

Marciniak et al. (2008)

Szabo (1998)

Weidenschilling et al. (1990)

\section{(80) Sappho}

Burchi et al. (1985)

Debehogne et al. (1990)

Harris \& Young (1983)

Scaltriti \& Zappala (1978)

USNO

\section{(82) Alkmene}

Harris et al. (1984)

USNO

\section{(87) Sylvia}

Blanco et al. (1989)

Harris \& Young (1980)

Prokof'eva et al. (1992)

Schober \& Surdej (1979)

Weidenschilling et al. (1987)

Weidenschilling et al. (1990)

\section{(88) Thisbe}

di Martino \& Cacciatori (1984a)

di Martino et al. (1987b)

Harris et al. (1992)

Choloniewski (1979)

Schober et al. (1979)

Weidenschilling et al. (1987)

Weidenschilling et al. (1990)

\section{(107) Camilla}

di Martino et al. (1987a)

Harris \& Young (1989)

Polishook (2009)

Weidenschilling et al. (1987)

Weidenschilling et al. (1990) 


\section{(110) Lydia}

Dotto et al. (1992)

Durech et al. (2007)

Lagerkvist et al. (1995)

Taylor et al. (1971)

\section{(115) Thyra}

Dotto et al. (1995)

Chang et al. (1981)

Magnusson \& Lagerkvist (1991)

McCheyne et al. (1985)

Michałowski et al. (2004)

Scaltriti et al. (1981)

\section{(121) Hermione}

Debehogne et al. (1978)

Descamps et al. (2009)

di Martino et al. (1987b)

Hutton (1990b)

Piironen et al. (1994)

\section{(125) Liberatrix}

Durech et al. (2007)

Hutton (1991)

Harris et al. (1992)

Lagerkvist et al. (1986)

Weidenschilling et al. (1987)

Weidenschilling et al. (1990)

Zappala et al. (1982)

\section{(129) Antigone}

Barucci et al. (1985)

di Martino \& Cacciatori (1984a)

di Martino et al. (1987b)

Dotto et al. (1992)

Scaltriti \& Zappala (1977b)

Torppa et al. (2003)

Vesely \& Taylor (1985)

Weidenschilling et al. (1987)

Weidenschilling et al. (1990)

\section{(130) Elektra}

Danforth \& Ratcliff (1994)

Debehogne et al. (1990)

Durech et al. (2007)

Harris \& Young (1989)

Harris et al. (1999)

Shevchenko et al. (1996)

Weidenschilling et al. (1987)

\section{(132) Aethra}

USNO

Weidenschilling et al. (1990)

Wisniewski et al. (1997)

\section{(135) Hertha}

Dotto et al. (1992)

Harris \& Young (1980)

Harris et al. (1992)

Lagerkvist (1981)

Lagerkvist et al. (1986)

Lagerkvist et al. (1995)
Tancredi \& Gallardo (1991)

Torppa et al. (2003)

\section{(146) Lucina}

Harris \& Young (1983)

Harris \& Young (1989)

Harris et al. (1999)

Piironen et al. (1998)

Schober (1983b)

USNO

(152) Atala

Schober (1983b)

USNO

(158) Koronis

Binzel (1987)

Slivan \& Binzel (1996)

Slivan et al. (2003)

(160) Una

di Martino et al. (1994a)

Harris et al. (1999)

Marciniak et al. (2009b)

(167) Urda

Slivan \& Binzel (1996)

Tedesco (1979)

USNO

Warner et al. (2008)

(182) Elsa

Harris et al. (1980)

Harris et al. (1992)

Pilcher et al. (2009)

USNO

\section{(184) Dejopeja}

Hutton (1995)

Marciniak et al. (2007)

Tedesco (1979)

(192) Nausikaa

Dotto et al. (1995)

Lagerkvist et al. (1995)

Scaltriti \& Zappala (1976)

Yang et al. (1965)

\section{(196) Philomela}

Durech et al. (2007)

Erikson et al. (1991)

Kryszczyǹska et al. (1996)

Yang et al. (1965)

Zappala et al. (1983b)

\section{(201) Penelope}

Harris \& Young (1989)

Lagerkvist et al. (1981)

Pfleiderer et al. (1987)

Surdej et al. (1983)

Weidenschilling et al. (1987)

Weidenschilling et al. (1990) 


\section{(208) Lacrimosa}

Binzel (1987)

Slivan \& Binzel (1996)

\section{(218) Bianca}

Carlsson \& Lagerkvist (1981)

Denchev et al. (1998)

Durech et al. (2007)

Harris \& Young (1989)

Kryszczyǹska et al. (1996)

\section{(230) Athamantis}

Harris \& Young (1980)

Harris \& Young (1989)

Harris et al. (1992)

Harris et al. (1999)

Yang et al. (1965)

Zeigler \& Florence (1985)

\section{(250) Bettina}

di Martino \& Cacciatori (1984a)

Dotto et al. (1992)

Lagerkvist \& Rickman (1981)

Lagerkvist et al. (1986)

Lagerkvist et al. (1995)

Weidenschilling et al. (1990)

\section{(276) Adelheid}

di Martino et al. (1995)

Marciniak et al. (2007)

Piironen et al. (1994)

\section{(277) Elvira}

Binzel (1987)

Slivan \& Binzel (1996)

Slivan et al. (2003)

\section{(278) Paulina}

Barucci et al. (1994)

USNO

\section{(283) Emma}

Fauerbach - unpublished data Michałowski et al. (2006)

Stanzel (1978)

\section{(306) Unitas}

Durech et al. (2007)

Hainaut-Rouelle et al. (1995)

Harris \& Young (1983)

Piironen et al. (1998)

\section{(311) Claudia}

Binzel (1987)

Slivan \& Binzel (1996)

Slivan et al. (2003)

\section{(321) Florentina}

\section{Frey (2000)}

Slivan \& Binzel (1996)

Slivan et al. (2003)
Thizy - unpublished data

van Houten-Groeneveld \& van Houten (1958)

Warner (2000)

\section{(349) Dembowska}

di Martino et al. (1987b)

Haupt (1980)

Chang \& Chang (1963)

McCheyne et al. (1985)

Weidenschilling et al. (1987)

Zappala et al. (1979)

\section{(350) Ornamenta}

Marciniak et al. (2009a)

Schober et al. (1993)

\section{(360) Carlova}

di Martino et al. (1987a)

Dotto et al. (1995)

Harris \& Young (1983)

USNO

\section{(377) Campania}

di Martino et al. (1994a)

Hainaut-Rouelle et al. (1995)

Lagerkvist et al. (1987)

Marciniak et al. (2008)

Schober et al. (1994)

Tedesco (1979)

\section{(378) Holmia}

di Martino et al. (1994b)

Marciniak et al. (2008)

\section{(382) Dodona}

di Martino (1986)

Lagerkvist et al. (1986)

Michałowski et al. (2004)

\section{(409) Aspasia}

di Martino \& Cacciatori (1984b)

Hainaut-Rouelle et al. (1995)

Hanslmeier (1981)

Lagerkvist (1981)

Piironen et al. (1998)

USNO

Warner et al. (2008)

\section{(416) Vaticana}

Durech et al. (2009)

Erikson et al. (1991)

Lagerkvist et al. (1987)

Miles (1990)

Schober et al. (1994)

USNO

\section{(423) Diotima}

di Martino \& Cacciatori (1984b)

Dotto et al. (1995)

Durech et al. (2007)

Hutton (1990a) 
Hainaut-Rouelle et al. (1995)

Schober (1983a)

\section{(484) Pittsburghia}

Binzel (1987)

USNO

\section{(511) Davida}

di Martino et al. (1987a)

Gehrels \& Owings (1962)

Groeneveld \& Kuiper (1954a)

Chang \& Chang (1963)

Vesely \& Taylor (1985)

Weidenschilling et al. (1987)

\section{(516) Amherstia}

Dotto et al. (1992)

Harris \& Young (1980)

Lagerkvist et al. (1987)

USNO

\section{(532) Herculina}

Cunnigham (1983)

Drummond et al. (1985)

Erikson et al. (1991)

Groeneveld \& Kuiper (1954b)

Harris \& Young (1979)

Chang \& Chang (1963)

Kaasalainen et al. (2002b)

Lebofsky et al. (1988)

Michałowski et al. (1995)

Shevchenko et al. (1992)

Taylor et al. (1987)

Weidenschilling et al. (1990)

\section{(534) Nassovia}

Binzel (1987)

Slivan \& Binzel (1996)

Tedesco (1979)

\section{(556) Phyllis}

Harris et al. (1992)

Marciniak et al. (2007)

Zappala et al. (1983b)

\section{(584) Semiramis}

Hainaut-Rouelle et al. (1995)

Harris et al. (1992)

Magnusson (1990)

Weidenschilling et al. (1987)

Weidenschilling et al. (1990)

\section{(595) Polyxena}

Hainaut-Rouelle et al. (1995)

USNO

Warner (2008)

\section{(614) Pia}

di Martino et al. (1994b)

USNO

\section{(624) Hektor}

Dahlgren et al. (1991)

Detal et al. (1994)

Dunlap \& Gehrels (1969)

Hainaut-Rouelle et al. (1995)

Hartmann \& Cruikshank (1978)

\section{(628) Christine}

Stephens (2003)

USNO

\section{(665) Sabine}

Hainaut-Rouelle et al. (1995)

Michałowski et al. (2006)

Riccioli et al. (1995)

\section{(675) Ludmilla}

Bernasconi - unpublished data Chang et al. (1981)

Schober \& Dvorak (1975)

Velichko et al. (1995)

\section{(690) Wratislavia}

Denchev (2000, private comm.)

Hutton (1988)

Hutton (1995)

Michałowski et al. (2006)

\section{(694) Ekard}

Hainaut-Rouelle et al. (1995)

Weidenschilling et al. (1987)

Weidenschilling et al. (1990)

Zeigler \& Florence (1985)

\section{(714) Ulula}

Schober \& Stadler (1990) USNO

\section{(720) Bohlinia}

Binzel (1987)

Slivan \& Binzel (1996)

Slivan et al. (2003)

\section{(747) Winchester}

Harris \& Young (1980)

Marciniak et al. (2009b)

Vesely \& Taylor (1985)

Zappala et al. (1983b)

(770) Bali

USNO

Wisniewski et al. (1997)

\section{(771) Libera}

Binzel (1987)

Marciniak et al. (2009a)

Warner (2000)

\section{(776) Berbericia}

Debehogne et al. (1983)

di Martino et al. (1987b) 
Ďurech et al. (2007)

Hainaut-Rouelle et al. (1995)

Harris et al. (1992)

Schober (1979)

\section{(849) Ara}

Harris et al. (1992)

Marciniak et al. (2009b)

\section{(915) Cosette}

di Martino et al. (1994b) USNO

\section{(984) Gretia}

di Martino (1984)

Marciniak et al. (2009a)

Piironen et al. (1994)

van Houten (1962)

\section{(1012) Sarema}

Binzel (1987)

USNO

\section{(1022) Olympiada}

USNO

Warner et al. (2008)

\section{(1036) Ganymed}

Hahn et al. (1989)

Lupishko et al. (1987b)

\section{(1088) Mitaka}

\section{USNO}

Wisniewski et al. (1997)

\section{(1223) Neckar}

Binzel (1987)

Michałowski et al. (2000)

Slivan \& Binzel (1996)

Tedesco (1979)

\section{(1270) Datura}

Székely et al. (2005)

Vokrouhlický et al. (2009)

Wisniewski et al. (1997)

\section{(1580) Betulia}

Erikson et al. (1991)

Lagerkvist (1977)

Tedesco et al. (1978)

Wisniewski et al. (1997)

\section{(1600) Vyssotsky}

Higgins (2008)

Warner (1999)

Warner et al. (2006)

\section{(1620) Geographos}

Dunlap (1974)

Ďurech et al. (2008a)

Magnusson et al. (1996)
Michałowski et al. (1994)

Weidenschilling et al. (1990)

\section{(1627) Ivar}

Hahn et al. (1989)

Hoffmann \& Geyer (1990)

Chernova et al. (1995)

Kaasalainen et al. (2004)

Pravec et al. (1996)

Velichko et al. (1990)

\section{(1862) Apollo}

Ďurech et al. (2008b)

Hahn (1983)

Harris et al. (1987)

Kaasalainen et al. (2007)

\section{(1980) Tezcatlipoca}

Kaasalainen et al. (2004)

Wisniewski et al. (1997)

\section{(2100) Ra-Shalom}

Harris et al. (1992)

Kaasalainen et al. (2004)

Ostro et al. (1984)

Pravec et al. (1998)

\section{(3908) Nyx}

Drummond \& Wisniewski (1990)

Kaasalainen et al. (2004)

\section{(5587) $1990 \mathrm{SB}$}

Kaasalainen et al. (2004)

Koff et al. (2002)

Polishook (2009)

Pravec et al. (1998)

\section{(6053) 1993 BW3}

Pravec et al. (1997)

\section{References}

Ahmad, I. I. 1954, ApJ, 120, 551

Barucci, M. A. 1983, A\&AS, 54, 471

Barucci, M. A., Fulchignoni, M., Burchi, R., et al. 1985, Icarus, 61, 152

Barucci, M. A., Bockelee-Morvan, D., Lecacheux, J., Brahic, A., \& Clairemidi, S. 1986, A\&A, 163, 261

Barucci, M. A., di Martino, M., Dotto, E., et al. 1994, Icarus, 109, 267

Belskaya, I. N., Dovgopol, A. N., Erikson, A., Lagerkvist, C.-I., \& Oja, T. 1993, A\&AS, 101, 507

Berthier, J., Hestroffer, D., Carry, B., et al. 2008, LPI Contributions, 1405, 8374

Binzel, R. P. 1984, Icarus, 59, 456

Binzel, R. P. 1987, Icarus, 72, 135

Birch, P. V., \& Taylor, R. C. 1989, A\&AS, 81, 409

Birch, P. V., Tedesco, E. F., Taylor, R. C., et al. 1983, Icarus, 54, 1

Blanco, C., di Martino, M., Gonano, M., Jaumann, R., \& Mottola, S. 1989, Mem.

Soc. Astron. Itali., 60, 195

Burchi, R. 1972, Mem. Soc. Astron. Ital., 43, 27

Burchi, R., \& Milano, L. 1974, A\&AS, 15, 173

Burchi, R., \& Milano, L. 1983, Moon and Planets, 28, 17

Burchi, R., D’Ambrosio, V., Tempesti, P., et al. 1985, A\&AS, 60, 9

Carlsson, M., \& Lagerkvist, C.-I. 1981, A\&AS, 44, 15

Carlsson, M., \& Lagerkvist, C.-I. 1983, A\&AS, 53, 157

Chang, Y. C., \& Chang, C. S. 1962, Acta Astron. Sinica, 10, 101

Chang, Y. C., \& Chang, C. S. 1963, Acta Astron. Sinica, 11, 139

Chang, Y. C., Wan-Tsing, H., \& Fu-Yuan, K. 1959, Acta Astron. Sinica, 7, 204 
Chang, Y. C., Zhou, X.-H., Yang, X.-Y., et al. 1981, Acta Astron. Sinica, 22, 169 Chernova, G. P., Kiselev, N. N., Krugley, Y. N., et al. 1995, AJ, 110, 1875 Choloniewski, J. 1979, Acta Astron., 29, 105

Cunnigham, C. J. 1983, Minor Planet Bulletin, 10, 26

Dahlgren, M., Lagerkvist, C.-I., Fitzsimmons, A., et al. 1991, MNRAS, 250, 115

Danforth, C. W., \& Ratcliff, S. J. 1994, Minor Planet Bulletin, 21, 1

Debehogne, H., \& Zappala, V. 1980, A\&AS, 40, 257

Debehogne, H., Surdej, A., \& Surdej, J. 1978, A\&AS, 32, 127

Debehogne, H., de Sanctis, G., \& Zappala, V. 1983, Icarus, 55, 236

Debehogne, H., Lagerkvist, C.-I., Magnusson, P., et al. 1990, in Asteroids, Comets, Meteors III, ed. C. I. Lagerkvist, H. Rickman, \& B. A. Lindblad, 45

Delbo, M., \& Tanga, P. 2009, Planet. Space Sci., 57, 259

Denchev, P., Magnusson, P., \& Donchev, Z. 1998, Planet. Space Sci., 46, 673

Descamps, P., Marchis, F., Durech, J., et al. 2009, Icarus, 203, 88

Detal, A., Hainaut, O., Pospieszalska-Surdej, A., et al. 1994, A\&A, 281, 269

di Martino, M. 1984, Icarus, 60, 541

di Martino, M. 1986, in Asteroids, Comets, Meteors II, ed. C.-I. Lagerkvist,

H. Rickman, B. A. Lindblad, \& H. Lundstedt, 81

di Martino, M., \& Cacciatori, S. 1984a, Icarus, 60, 75

di Martino, M., \& Cacciatori, S. 1984b, A\&A, 130, 206

di Martino, M., Zappala, V., de Campos, J. A., Debehogne, H., \& Lagerkvist,

C.-I. 1987a, A\&AS, 67, 95

di Martino, M., Zappala, V., de Sanctis, G., et al. 1987b, Icarus, 69, 338

di Martino, M., Zappala, V., Cellino, A., et al. 1989, A\&A, 223, 352

di Martino, M., Blanco, C., Riccioli, D., et al. 1994a, Icarus, 107, 269

di Martino, M., Dotto, E., Barucci, M. A., Fulchignoni, M., \& Rotundi, A. 1994b, Icarus, 109, 210

di Martino, M., Dotto, E., Cellino, A., Barucci, M. A., \& Fulchignoni, M. 1995, A\&AS, 112, 1

Dotto, E., Barucci, M. A., Fulchignoni, M., et al. 1992, A\&AS, 95, 195

Dotto, E., de Angelis, G., di Martino, M., et al. 1995, Icarus, 117, 313

Drummond, J. D., \& Wisniewski, W. Z. 1990, Icarus, 83, 349

Drummond, J. D., Hege, E. K., Cocke, W. J., et al. 1985, Icarus, 61, 232

Dunlap, J. L. 1974, AJ, 79, 324

Dunlap, J. L., \& Gehrels, T. 1969, AJ, 74, 796

Durech, J., \& Kaasalainen, M. 2003, A\&A, 404, 709

Durech, J., Grav, T., Jedicke, R., Kaasalainen, M., \& Denneau, L. 2005, Earth, Moon, and Planets, 97, 179

Ďurech, J., Kaasalainen, M., Marciniak, A., et al. 2007, A\&A, 465, 331

Ďurech, J., Vokrouhlický, D., Kaasalainen, M., et al. 2008a, A\&A, 489, L25

Durech, J., Vokrouhlický, D., Kaasalainen, M., et al. 2008b, A\&A, 488, 345

Durech, J., Kaasalainen, M., Warner, B. D., et al. 2009, A\&A, 493, 291

Erikson, A., Lagerkvist, C.-I., Lindgren, M., et al. 1991, A\&AS, 91, 259

Foglia, S. 1992, Minor Planet Bulletin, 19, 19

Foglia, S. 1995, Minor Planet Bulletin, 22, 36

Frey, G. 2000, Minor Planet Bulletin, 27, 29

Gehrels, T. 1956, ApJ, 123, 331

Gehrels, T., \& Owings, D. 1962, ApJ, 135, 906

Gehrels, T., \& Taylor, R. C. 1977, AJ, 82, 229

Groeneveld, I., \& Kuiper, G. P. 1954a, ApJ, 120, 200

Groeneveld, I., \& Kuiper, G. P. 1954b, ApJ, 120, 529

Hahn, G. 1983, in Asteroids, comets, meteors; Proceedings of the Meeting,

Uppsala, Sweden, June 20-22, ed. C.-I. Lagerkvist, \& H. Rickman, (A8526851 11-89), Uppsala, Sweden, Astronomiska Observatoriet, 35,

Hahn, G., Magnusson, P., Harris, A. W., et al. 1989, Icarus, 78, 363

Hainaut-Rouelle, M.-C., Hainaut, O. R., \& Detal, A. 1995, A\&AS, 112, 125

Hanslmeier, A. 1981, Mitt. Univ. Graz., 85

Hapke, B. 1981, J. Geophys. Res., 86, 3039

Hapke, B. 1984, Icarus, 59, 41

Hapke, B. 1986, Icarus, 67, 264

Harris, A. W., \& Young, J. 1979, Icarus, 38, 100

Harris, A. W., \& Young, J. W. 1980, Icarus, 43, 20

Harris, A. W., \& Young, J. W. 1983, Icarus, 54, 59

Harris, A. W., \& Young, J. W. 1989, Icarus, 81, 314

Harris, A. W., Young, J. W., Scaltriti, F., et al. 1980, Icarus, 41, 316

Harris, A. W., Young, J. W., Scaltriti, F., et al. 1984, Icarus, 57, 251

Harris, A. W., Young, J. W., Goguen, J., et al. 1987, Icarus, 70, 246

Harris, A. W., Young, J. W., Bowell, E., et al. 1989a, Icarus, 77, 171

Harris, A. W., Young, J. W., Contreiras, L., et al. 1989b, Icarus, 81, 365

Harris, A. W., Young, J. W., Dockweiler, T., et al. 1992, Icarus, 95, 115

Harris, A. W., Young, J. W., Bowell, E., et al. 1999, Icarus, 142, 173

Hartmann, W. K., \& Cruikshank, D. P. 1978, Icarus, 36, 353

Haupt, H. 1980, Mitt. Univ. Graz., 69

Haupt, H., \& Hanslmeier, A. 1985, Mitt. der Univ. Sternwarte Graz., 107

Higgins, D. 2008, Minor Planet Bulletin, 35, 30

Hoffmann, M., \& Geyer, E. H. 1990, Acta Astron., 40, 389

Hollis, A. J., Bembrick, C. S., Dumont, M., et al. 1987, Journal of the British
Astronomical Association, 97, 220

Hutton, R. G. 1988, Minor Planet Bulletin, 15, 21

Hutton, R. G. 1989, Minor Planet Bulletin, 16, 16

Hutton, R. G. 1990a, Boletin de la Asociacion Argentina de Astronomia La Plata Argentina, 36, 193

Hutton, R. G. 1990b, Minor Planet Bulletin, 17, 41

Hutton, R. G. 1991, Minor Planet Bulletin, 18, 39

Hutton, R. G. 1995, Rev. Mex. Astron. Astrofis, 31, 19

Hutton, R. G., \& Blain, A. 1988, Minor Planet Bulletin, 15, 3

Kaasalainen, M. 2009, Inverse Problems and Imaging, submitted

Kaasalainen, M., \& Lamberg, L. 2006, Inverse Problems, 22, 749

Kaasalainen, M., \& Torppa, J. 2001, Icarus, 153, 24

Kaasalainen, M., Torppa, J., \& Muinonen, K. 2001, Icarus, 153, 37

Kaasalainen, M., Mottola, S., \& Fulchignomi, M. 2002a, in Asteroids III, ed.

W. F. Bottke, A. Cellino, P. Paolicchi, \& R. P. Binzel (Tucson: University of Arizona Press), 139

Kaasalainen, M., Torppa, J., \& Piironen, J. 2002b, Icarus, 159, 369

Kaasalainen, M., Pravec, P., Krugly, Y. N., et al. 2004, Icarus, 167, 178

Kaasalainen, S., Kaasalainen, M., \& Piironen, J. 2005, A\&A, 440, 1177

Kaasalainen, M., Ďurech, J., Warner, B. D., Krugly, Y. N., \& Gaftonyuk, N. M. 2007, Nature, 446, 420

Koff, R. A., Pravec, P., Šarounová, L., et al. 2002, Minor Planet Bulletin, 29, 51

Kryszczyǹska, A., Colas, F., Berthier, J., Michałowski, T., \& Pych, W. 1996, Icarus, 124, 134

Kryszczyńska, A., La Spina, A., Paolicchi, P., et al. 2007, Icarus, 192, 223

Lagerkvist, C.-I. 1977, Icarus, 32, 233

Lagerkvist, C.-I. 1981, A\&AS, 44, 345

Lagerkvist, C.-I., \& Rickman, H. 1981, Moon and Planets, 24, 437

Lagerkvist, C.-I., \& Williams, I. P. 1987, A\&AS, 68, 295

Lagerkvist, C.-I., Rickman, H., Scaltriti, F., et al. 1981, A\&A, 104, 148

Lagerkvist, C.-I., Hahn, G., Magnusson, P., Rickman, H., \& Hammarback, G. 1986, in Asteroids, Comets, Meteors II, ed. C.-I. Lagerkvist, H. Rickman, B. A. Lindblad, \& H. Lundstedt, 67

Lagerkvist, C.-I., Hahn, G., Magnusson, P., et al. 1987, A\&AS, 70, 21

Lagerkvist, C.-I., Magnusson, P., Williams, I. P., Buontempo, M. E., \& Gibbs, P. 1988, A\&AS, 73, 395

Lagerkvist, C.-I., Magnusson, P., Williams, I. P., et al. 1989, A\&AS, 78, 519

Lagerkvist, C.-I., Erikson, A., Debehogne, H., et al. 1995, A\&AS, 113, 115

Lamy, P. L., Kaasalainen, M., Lowry, S., et al. 2008, A\&A, 487, 1179

Lebofsky, L. A., Greenberg, R., Tedesco, E. F., et al. 1988, Icarus, 75, 518

Lupishko, D. F., Kiselev, N. N., \& Chernova, G. P. 1979, Pisma Astronomicheskii Zhurnal, 5, 201

Lupishko, D. F., Kiselev, N. N., Chernova, G. P., et al. 1980, Pisma Astronomicheskii Zhurnal, 6, 184

Lupishko, D. F., Tupieva, F. A., Velichko, F. P., Kiselev, N. N., \& Chernova, G. P. 1981, Astronomicheskii Vestnik, 15, 25

Lupishko, D. F., Belskaya, I. N., Tupieva, F. A., et al. 1982, Astronomicheskii Vestnik, 16, 101

Lupishko, D. F., Belskaya, I. N., \& Tupieva, F. A. 1983, Pisma Astronomicheskii Zhurnal, 9, 691

Lupishko, D. F., Velichko, F. P., Belskaya, I. N., et al. 1987a, Kinematika i Fizika Nebesnykh Tel, 3, 36

Lupishko, D. F., Velichko, F. P., Kazakov, V. V., et al. 1987b, Kinematika i Fizika Nebesnykh Tel, 3, 92

Lupishko, D. F., Tupieva, F. A., Velichko, F. P., et al. 1989, Bjull. Inst. Astrofizikii, 80, 13

Lustig, G., \& Dvorak, R. 1975, Acta Physica Austriaca, 43, 89

Lustig, G., \& Hahn, G. 1976, Acta Physica Austriaca, 44, 199

Magnusson, P. 1990, Icarus, 85, 229

Magnusson, P., \& Lagerkvist, C.-I. 1991, A\&AS, 87, 269

Magnusson, P., Barucci, M. A., Drummond, J. D., et al. 1989, in Asteroids II, ed. R. P. Binzel, T. Gehrels, \& M. S. Matthews (Tucson: University of Arizona Press), 66

Magnusson, P., Dahlgren, M., Barucci, M. A., et al. 1996, Icarus, 123, 227

Marciniak, A., Michałowski, T., Kaasalainen, M., et al. 2007, A\&A, 473, 633

Marciniak, A., Michałowski, T., Kaasalainen, M., et al. 2008, A\&A, 478, 559

Marciniak, A., Michałowski, T., Hirsch, R., et al. 2009a, A\&A, 508, 1503

Marciniak, A., Michałowski, T., Hirsch, R., et al. 2009b, A\&A, 498, 313

McCheyne, R. S., Eaton, N., Green, S. F., et al. 1984, Icarus, 59, 286

McCheyne, R. S., Eaton, N., \& Meadows, A. J. 1985, Icarus, 61, 443

Melillo, F. J. 1986, Minor Planet Bulletin, 13, 21

Melillo, F. J. 1987a, Minor Planet Bulletin, 14, 42

Melillo, F. J. 1987b, Minor Planet Bulletin, 14, 21

Michałowski, T., \& Velichko, F. P. 1990, Acta Astron., 40, 321

Michałowski, T., Kwiatkowski, T., Borczyk, W., et al. 1994, Acta Astron., 44, 223

Michałowski, T., Velichko, F. P., di Martino, M., et al. 1995, Icarus, 118, 292

Michałowski, T., Pych, W., Berthier, J., et al. 2000, A\&AS, 146, 471 
Michałowski, T., Kwiatkowski, T., Kaasalainen, M., et al. 2004, A\&A, 416, 353 Michałowski, T., Kaasalainen, M., Polińska, M., et al. 2006, A\&A, 459, 663

Mignard, F., Cellino, A., Muinonen, K., et al. 2007, Earth Moon and Planets, 101,97

Miles, R. 1990, Minor Planet Bulletin, 17, 25

Millis, R. L., Wasserman, L. H., Bowell, E., et al. 1981, AJ, 86, 306

Müller, T. G., Sekiguchi, T., Kaasalainen, M., Abe, M., \& Hasegawa, S. 2005, A\&A, 443, 347

Neely, A. W. 1992, Minor Planet Bulletin, 19, 28

Ostro, S. J., Harris, A. W., Campbell, D. B., Shapiro, I. I., \& Young, J. W. 1984, Icarus, 60, 391

Pavlovski, K., Knezevic, Z., \& Muminovic, M. 1981, Publ. Astron. Obs. Sarajevo, 1, 1, 225

Pfleiderer, J., Pfleiderer, M., \& Hanslmeier, A. 1987, A\&AS, 69, 117

Piironen, J., Bowell, E., Erikson, A., et al. 1994, A\&AS, 106, 587

Piironen, J., Lagerkvist, C.-I., Erikson, A., et al. 1998, A\&AS, 128, 525

Piironen, J. O., Poutanen, M., di Martino, M., et al. 1985, A\&AS, 61, 299

Pilcher, F. 2009, Minor Planet Bulletin, 36, 133

Pilcher, F., Benishek, V., \& Krajewski, R. 2009, Minor Planet Bulletin, 36, 40

Polishook, D. 2009, Minor Planet Bulletin, 36, 119

Pravec, P., Šarounová, L., \& Wolf, M. 1996, Icarus, 124, 471

Pravec, P., Wolf, M., \& Šarounová, L. 1998, Icarus, 136, 124

Pravec, P., Wolf, M., Šarounová, L., Harris, A. W., \& Davies, J. K. 1997, Icarus, 127,441

Pravec, P., Harris, A. W., \& Michałowski, T. 2002, in Asteroids III, ed. W. F. Bottke, A. Cellino, P. Paolicchi, \& R. P. Binzel (Tucson: University of Arizona Press), 113

Prokof'eva, V. V., Demchik, M. I., Karachkina, L. G., et al. 1992, Astronomicheskii Vestnik, 26, 3

Riccioli, D., Blanco, C., di Martino, M., et al. 1995, A\&AS, 111, 297

Sather, R. E. 1976, AJ, 81, 67

Scaltriti, F., \& Zappala, V. 1975, A\&AS, 19, 249

Scaltriti, F., \& Zappala, V. 1976, A\&AS, 23, 167

Scaltriti, F., \& Zappala, V. 1977a, Icarus, 31, 498

Scaltriti, F., \& Zappala, V. 1977b, A\&A, 56, 7

Scaltriti, F., \& Zappala, V. 1977c, A\&AS, 30, 169

Scaltriti, F., \& Zappala, V. 1978, Icarus, 34, 428

Scaltriti, F., Zappala, V., \& Stanzel, R. 1978, Icarus, 34, 93

Scaltriti, F., Zappala, V., \& Harris, A. W. 1981, Icarus, 46, 275

Schober, H. J. 1976, A\&A, 53, 115

Schober, H. J. 1978, A\&AS, 34, 377

Schober, H. J. 1979, A\&AS, 38, 91

Schober, H. J. 1983a, A\&A, 127, 301

Schober, H. J. 1983b, A\&AS, 53, 71

Schober, H. J., \& Dvorak, R. 1975, A\&A, 44, 81

Schober, H. J., \& Stadler, M. 1990, A\&A, 230, 233

Schober, H. J., \& Surdej, J. 1979, A\&AS, 38, 269

Schober, H. J., Scaltriti, F., \& Zappala, V. 1979, A\&AS, 36,

Schober, H. J., Erikson, A., Hahn, G., Lagerkvist, C. I., \& Oja, T. 1993, A\&AS 101,499

Schober, H. J., Erikson, A., Hahn, G., et al. 1994, A\&AS, 105, 281

Schroll, A., Haupt, H. F., \& Maitzen, H. M. 1976, Icarus, 27, 147

Schroll, A., Schober, H. J., \& Lagerkvist, C. I. 1981, A\&A, 104, 296

Seidelmann, P. K., Archinal, B. A., A'Hearn, M. F., et al. 2007, Celestial Mechanics and Dynamical Astronomy, 98, 155

Shatzel, A. V. 1954, ApJ, 120, 547

Shevchenko, V. G., Cherny, V. G., Krugly, Y. N., et al. 1992, Icarus, 100, 295

Shevchenko, V. G., Chiorny, V. G., Kalashnikov, A. V., et al. 1996, A\&AS, 115, 475

Shevchenko, V. G., Krugly, Y. N., Lupishko, D. F., Harris, A. W., \& Chernova,

G. P. 1993, Astronomicheskii Vestnik, 27, 75

Slivan, S. M., \& Binzel, R. P. 1996, Icarus, 124, 452
Slivan, S. M., Binzel, R. P., Crespo da Silva, L. D., et al. 2003, Icarus, 162, 285 Stanzel, R. 1978, A\&AS, 34, 373

Stephens, R. D. 2003, Minor Planet Bulletin, 30, 69

Surdej, J., Louis, B., Cramer, N., et al. 1983, A\&AS, 54, 371

Surdej, J., Pospieszalska-Surdej, A., Michałowski, T., et al. 1986, A\&A, 170, 167

Szabo, G. 1998, Acta Physica Polonica A, 93, 23

Székely, P., Kiss, L. L., Szabó, G. M., et al. 2005, Planet. Space Sci., 53, 925

Tancredi, G., \& Gallardo, T. 1991, A\&A, 242, 279

Taylor, R. C. 1977, AJ, 82, 441

Taylor, R. C. 1978, AJ, 83, 201

Taylor, R. C., \& Tedesco, E. F. 1983, Icarus, 54, 13

Taylor, R. C., Gehrels, T., \& Silvester, A. B. 1971, AJ, 76, 141

Taylor, R. C., Gehrels, T., \& Capen, R. C. 1976, AJ, 81, 778

Taylor, R. C., Birch, P. V., Drummond, J., Pospieszalska-Surdej, A., \& Surdej, J. 1987, Icarus, 69, 354

Taylor, R. C., Birch, P. V., Pospieszalska-Surdej, A., et al. 1988, Icarus, 73, 314

Tedesco, E., Drummond, J., Candy, M., et al. 1978, Icarus, 35, 344

Tedesco, E. F. 1979, Ph.D. Thesis, A\&A (New Mexico State Univ., University Park)

Tedesco, E. F., \& Sather, R. E. 1981, AJ, 86, 1553

Tedesco, E. F., \& Taylor, R. C. 1985, Icarus, 61, 241

Tedesco, E. F., Taylor, R. C., Drummond, J., et al. 1983, Icarus, 54, 30

Torppa, J., Kaasalainen, M., Michałowski, T., et al. 2003, Icarus, 164, 346

van Houten, C. J. 1962, Bull. Astron. Inst. Netherlands, 16, 160

van Houten-Groeneveld, I. 1981, A\&A, 98, 203

van Houten-Groeneveld, I., \& van Houten, C. J. 1958, ApJ, 127, 253

van Houten-Groeneveld, I., van Houten, C. J., \& Zappala, V. 1979, A\&AS, 35, 223

Velichko, F. P., Krugly, Y. N., Lupishko, D. F., et al. 1990, Astronomicheskij Tsirkulyar, 1546, 39

Velichko, F. P., Michalowski, T., Erikson, A., et al. 1995, A\&AS, 110, 125

Vesely, C. D., \& Taylor, R. C. 1985, Icarus, 64, 37

Veverka, J. 1971, Icarus, 15, 454

Vokrouhlický, D., Ďurech, J., Michałowski, T., et al. 2009, A\&A, 507, 495

Wamsteker, W., \& Sather, R. E. 1974, AJ, 79, 1465

Warner, B. 2000, Minor Planet Bulletin, 27, 4

Warner, B. D. 1999, Minor Planet Bulletin, 26, 31

Warner, B. D. 2008, Minor Planet Bulletin, 35, 171

Warner, B. D., Pray, D. P., Dyvig, R., et al. 2006, Minor Planet Bulletin, 33, 45

Warner, B. D., Durech, J., Fauerbach, M., et al. 2008, Minor Planet Bulletin, 35, 167

Weidenschilling, S. J., Chapman, C. R., Davis, D. R., et al. 1987, Icarus, 70, 191

Weidenschilling, S. J., Chapman, C. R., Davis, D. R., Greenberg, R., \& Levy, D. H. 1990, Icarus, 86, 402

Wisniewski, W. Z., Michałowski, T. M., Harris, A. W., et al. 1997, Icarus, 126, 395

Wood, X. H. J., \& Kuiper, G. P. 1963, ApJ, 137, 1279

Yang, X. Y., Zhang, Y. Y., \& Li, X.-Q. 1965, Acta Astron. Sinica, 13, 66

Zappala, V., \& van Houten-Groeneveld, I. 1979, Icarus, 40, 289

Zappala, V., van Houten-Groeneveld, I., \& van Houten, C. J. 1979, A\&AS, 35 , 213

Zappala, V., Debehogne, H., Lagerkvist, C.-I., et al. 1982, A\&AS, 50, 23

Zappala, V., di Martino, M., \& Cacciatori, S. 1983a, Icarus, 56, 319

Zappala, V., Scaltriti, F., \& di Martino, M. 1983b, Icarus, 56, 325

Zappala, V., di Martino, M., Knezevic, Z., et al. 1984, A\&A, 130, 208

Zeigler, K. W., \& Florence, W. B. 1985, Icarus, 62, 512

Zhou, X.-H., \& Yang, X.-Y. 1981, Acta Astron. Sinica, 22, 378

Zhou, X. H., Yang, X. Y., \& Wu, Z. X. 1982, Acta Astron. Sinica, 23, 349

Ziegler, K. W., \& Florence, W. B. 1985, Minor Planet Bulletin, 12, 21 